\title{
Improvement in Storage Stability of Fish Fillets Using Dietary Soybean
}

\section{Phosphatidylcholine and Phosphatidylethanolamine}

\author{
Yoshihiro Murano", Tomoko FunABASHI and Hiroyuki TAKEUCHI \\ Central Research Laboratory of The Nisshin OilliO Group, Ltd., 1 Shinmei-cho, Yokosuka, Kanagawa 239-0832, Japan
}

Received May 27, 2008; Accepted December 15, 2008

We previously reported that soybean phospholipids improved the storage stability of fish fillets. The object of this study was to identify the active component of soybean phospholipids and its mechanism. Rainbow trout were fed purified diets supplemented with no phospholipids (Control), $1 \%$ soybean phosphatidylcholine (S-PC), and 1\% soybean phosphatidylethanolamine (S-PE) for 4 weeks. After the feeding period, fish fillets were subjected to an oxidation test. The storage stability of fish fillets was improved in the S-PC and S-PE groups compared to the Control group. The rate of phospholipid-bound docosahexaenoic acid (DHA) in the S-PC and S-PE groups was higher than that in the Control group. These results indicate that phosphatidylcholine and phosphatidylethanolamine in soybean phospholipids contributed to the improved storage stability of fish fillets. It was speculated that high phosphatidylcholine- and phosphatidylethanolamine-bound DHA levels were involved in the improved storage stability of fish fillets.

Keywords: soybean phospholipids, phosphatidylcholine, phosphatidylethanolamine, oxidation, fish

\section{Introduction}

N-3 polyunsaturated fatty acids (PUFAs), such as $\alpha$-linolenic acid, eicosapentaenoic acid (EPA), and docosahexaenoic acid (DHA), have been attracting attention regarding their preventive effects against diseases such as atherosclerosis, coronary arterial heart diseases, and hypertension (Conner, 2000; Takeuchi et al., 2007). N-3 PUFAs are easily oxidized because they contain many double bonds. We previously reported that soybean phospholipid administration inhibited lipid oxidation in fish fillets (Murano et al., 2008). This finding is of interest as a new technique to improve the storage stability of muscle food containing n-3 PUFAs.

In addition to the main components, phosphatidylcholine (PC) and phosphatidylethanolamine (PE), soybean phospholipids also contain other phospholipids, such as phosphatidylinositol and phosphatidylserine, with these other components accounting for about 55\% (Schneider, 2006). The first objective of this study was to confirm the effects of the main components of soybean phospholipids, soybean PC and soybean PE, and identify the active components that increase the storage stability of fish fillets. Rainbow trout were fed

*To whom correspondence should be addressed.

E-mail: y-murano@nisshin-oillio.com purified diets supplemented with soybean oil (Control), highpurity soybean phosphatidylcholine (S-PC), and high-purity soybean phosphatidylethanolamine (S-PE) for 4 weeks, and the storage stability of fish fillets was compared among the 3 groups.

The mechanism responsible for improving fish fillet storage stability using soybean phospholipids has not been clarified. Although the enhanced storage stability of muscle food with a high content of antioxidative substances, such as total tocopherol and phospholipids, has been reported (Kim et al., 2006; Yamaguchi and Toyomizu, 1984), the feeding of soybean phospholipids did not alter the total tocopherol or phospholipid content of fish fillets (Murano et al., 2008). The second objective was to clarify the reason for the soybean phospholipid-induced increase in the storage stability of fish fillets. It has been reported that the oxidative stability of muscle food varied depending on the fatty acid species bound to phospholipids, a biomembrane component (Stanley, 1991; Meynier et al., 1999). We investigated the effects of high-purity dietary soybean PC and soybean PE on phospholipid-bound fatty acids in fish fillets.

\section{Materials and Methods}

Fish and feeding experiment We used a purified diet, 
Table 1. Experimental diet composition.

\begin{tabular}{lccc}
\hline & \multicolumn{3}{c}{ Treatment group } \\
\cline { 2 - 4 } & Control & S-PC & S-PE \\
\hline Casein & 50 & 50 & 50 \\
Dextrin & 20 & 20 & 20 \\
Corn starch & 9 & 9 & 9 \\
Mineral mix & 5 & 5 & 5 \\
Vitamin mix & 1 & 1 & 1 \\
Lipids & & & \\
Fish oil & 10 & 10 & 10 \\
Soybean oil & 5 & 4 & 4 \\
Soybean phosphatidylcholine & 0 & 1 & 0 \\
Soybean phospatidylethanolamine & 0 & 0 & 1 \\
\hline
\end{tabular}

the composition of which is shown in Table 1. Vitamins and minerals were combined according to the formulation reported by Kaushik et al. (1998) and Ogino et al. (1979). Three experimental diets were prepared: Control (no added phospholipids), S-PC (soybean PC added), and S-PE (soybean PE added). All diets were supplemented with $10 \mathrm{~g} / 100$ $\mathrm{g}$ fish oil, and 5, 4 and $4 \mathrm{~g} / 100 \mathrm{~g}$ soybean oil was added to the Control, S-PC, and S-PE diets, respectively. S-PC was prepared by adding $1 \mathrm{~g} / 100 \mathrm{~g}$ soybean PC, and S-PE by adding $1 \mathrm{~g} / 100 \mathrm{~g}$ soybean PE (both from Sigma-Aldrich Co., St. Louis, MO, USA). We previously reported that $1 \%$ soybean phospholipids improved the storage stability of fish fillets. Therefore, $1 \%$ soybean phospholipids were substituted with $1 \%$ phosphatidylcholine or $1 \%$ phosphatidylethanolamine in the present study. The soybean phospholipids added to prepare the S-PC and S-PE diets were of high-level purity, 99\% and $98 \%$, respectively. The fatty acid compositions of the total lipids of the 3 diets are shown in Table 2.

The test fish were maintained as previously reported (Murano et al., 2008). Juvenile rainbow trout (Onchorhynchus mykiss), purchased from a local seed supplier, were used for the feeding experiment. At the start of the feeding experiment, a total of 45 fish (each weighing $2.0 \mathrm{~g}$ on average) were divided into 3 groups (15 fish per group). The fish were biologically and biochemically analyzed after the feeding period.

Oxidation test The oxidation test was performed as previously reported (Murano et al., 2008). The fillets were cut and adjusted to a weight of $1.0 \pm 0.1 \mathrm{~g}$. The samples were oxidized by incubation at $4 \pm 0.5^{\circ} \mathrm{C}$ under fluorescent lighting for 3 days. The positions of individual samples in
Table 2. Fatty acid composition of the experimental diets

\begin{tabular}{|c|c|c|c|}
\hline & \multicolumn{3}{|c|}{ Treatment group } \\
\hline & Control & S-PC & S-PE \\
\hline & \multicolumn{3}{|c|}{$(\mathrm{g} / 100 \mathrm{~g})$} \\
\hline $12: 0$ & 0.1 & 0.1 & 0.1 \\
\hline $14: 0$ & 4.0 & 4.0 & 4.0 \\
\hline $15: 0$ & 0.3 & 0.3 & 0.3 \\
\hline $16: 0$ & 12.7 & 12.9 & 13.1 \\
\hline $16: 1$ & 4.9 & 4.9 & 4.9 \\
\hline $17: 0$ & 0.2 & 0.2 & 0.2 \\
\hline $18: 0$ & 3.1 & 3.1 & 3.0 \\
\hline $18: 1$ & 21.4 & 20.6 & 20.6 \\
\hline $18: 2(n-6)$ & 18.3 & 19.0 & 18.9 \\
\hline $18: 3(n-3)$ & 2.7 & 2.7 & 2.7 \\
\hline $20: 0$ & 0.3 & 0.2 & 0.2 \\
\hline $20: 1$ & 9.0 & 9.0 & 9.0 \\
\hline $20: 2(n-6)$ & 0.2 & 0.2 & 0.2 \\
\hline $22: 0$ & 0.6 & 0.6 & 0.6 \\
\hline $22: 1$ & 8.0 & 8.0 & 8.0 \\
\hline $20: 5(n-3)$ & 7.1 & 7.1 & 7.1 \\
\hline $24: 0$ & 0.4 & 0.4 & 0.4 \\
\hline $24: 1$ & 0.5 & 0.5 & 0.5 \\
\hline $22: 5(n-3)$ & 1.0 & 1.0 & 1.0 \\
\hline $22: 6(n-3)$ & 5.2 & 5.2 & 5.2 \\
\hline
\end{tabular}


the incubator were strictly standardized during the oxidation period. The degree of lipid oxidation in the fish fillets was measured before and after the oxidation test.

Biochemical analysis Lipids were extracted from the samples by the method reported by Folch et al. (1957). The phospholipid content, fatty acid composition, thiobarbituric acid reactive substance (TBARS), and tocopherol content were analyzed as previously reported (Murano et al., 2008). The extracted lipids were fractionated into triacylglycerol (TG) and phospholipids by thin layer chromatography with chloroform : methanol (9: 1) (Silica gel 70 Plate; Wako Pure Chemical Industries, Ltd., Osaka, Japan). The fractionated TG was extracted from resin and measured by weight (Murano et al., 2007). Total phospholipids, PC, and PE were analyzed using the method established by the Japan Oil Chemists' Society No. 2. 4. 11 (2003). Fatty acids at positions $s n-1$ and $s n-2$ in PC and PE were analyzed by the phospholipase A2 method (Wells and Hanahan, 1969). The fatty acid analysis was performed by gas chromatography (Murano et al., 2008) after the conversion of fatty acyl groups in the TG to their methyl esters by heating in a sealed tube at $100^{\circ} \mathrm{C}$ for $1 \mathrm{~h}$ with methanolic sulfuric acid under nitrogen. Gas chromatography was performed using an Agilent Technologies 6869 series (Agilent Technologies, Santa Clara, CA, USA) equipped with a flame-ionization detector with a capillary column (OMEGAWAX320; Supelco, Bellefonte, PA, USA). Tricosanoic acid was used as an internal standard in quantitative gas chromatographic analyses (Shantha and Ackman, 1990). Tocopherol concentration was measured by a modified procedure of Huang and Shaw (1994). Analysis was carried out by HPLC (YMC-Pack SIL A-012 column, YMC Co., Ltd., Kyoto, Japan; F1100 Fluorescence Spectrophotometer, Hitachi High-Technologies Corporation, Tokyo, Japan). The eluting solvent was hexane : isopropyl alcohol (100: 0.5 ) at flow rate of $2 \mathrm{~mL} / \mathrm{min}$.

Statistical analysis Data are expressed as mean $\pm \mathrm{SD}$. Significant differences between groups were determined by one-way ANOVA followed by Tukey's multiple comparison test. Differences with a $P$-value of $<0.05$ were considered significant. A statistical software package (Toukei version 2, Esumi, Tokyo, Japan) was used for all statistical analyses.

\section{Results}

The growth performance of the rainbow trout fed the 3 diets for 4 weeks is shown in Fig. 1 and Table 3. There were no significant differences in body weight, feed conversion efficiency, or liver weight among the 3 diet groups. Dietary soybean PC or soybean PE did not affect growth performance.

There were no significant differences in the total lipid, TG, or phospholipid content of fish fillets at the end of the feeding period among the 3 groups, nor were there significant differences in the PC, PE, tocopherol (Table 4), or the total fatty acid composition (Table 5).

To investigate oxidative stability, fish fillets were subjected to the oxidation test after completion of the feeding experiment. As a parameter of lipid oxidation in fish fillets, the TBARS level was measured before and after the oxidation test. No significant difference in the TBARS level was noted among the 3 groups before the test. After the oxidation

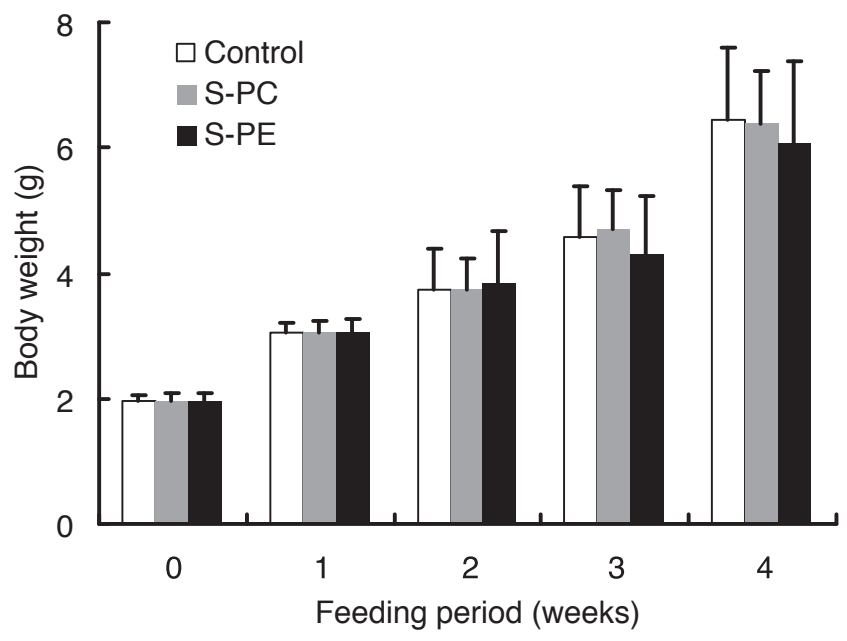

Fig. 1. Growth of rainbow trout during feeding period. Data are represented as mean $\pm \mathrm{SD}(\mathrm{n}=15)$.

Table 3. Growth and feed performance of rainbow trout after 4-week feeding period.

\begin{tabular}{lccccccccc}
\hline & \multicolumn{7}{c}{ Treatment group } \\
\cline { 2 - 12 } & \multicolumn{2}{c}{ Control } & \multicolumn{2}{c}{ S-PC } & \multicolumn{2}{c}{ S-PE } \\
\hline Initial body weight $(\mathrm{g})$ & 2.0 & \pm & 0.1 & 2.1 & \pm & 0.1 & 2.0 & \pm & 0.1 \\
Final body weight (g) & 6.5 & \pm & 1.1 & 6.4 & \pm & 0.9 & 6.1 & \pm & 1.3 \\
Feed conversion efficiency (\%) & 136 & & & 132 & & 124 & \\
Liver weight (g) & 0.14 & \pm & 0.04 & $0.14 \pm$ & 0.05 & 0.15 & \pm & 0.04 \\
\hline
\end{tabular}

Data are represented as mean $\pm \mathrm{SD}, \mathrm{n}=15$. 
Table 4. Fillet total lipid, triacylglycerol, phospholipid, and total tocopherol contents at the end of feeding.

\begin{tabular}{|c|c|c|c|}
\hline \multirow[b]{3}{*}{ Total lipids (g/100g) } & \multicolumn{3}{|c|}{ Treatment group } \\
\hline & Control & S-PC & S-PE \\
\hline & $5.6 \pm 1.2$ & $5.6 \pm 0.4$ & $5.5 \pm 1.0$ \\
\hline Triacylglycerol (g/100g) & $3.6 \pm 0.5$ & $3.5 \pm 0.5$ & $4.0 \pm 0.5$ \\
\hline Phospholipids (g/100g) & $1.0 \pm 0.1$ & $1.1 \pm 0.1$ & $1.2 \pm 0.1$ \\
\hline Phosphatidylcholine (g/100g) & $0.74 \pm 0.14$ & $0.75 \pm 0.03$ & $0.72 \pm 0.19$ \\
\hline Phosphatidylethanolamine (g/100g) & $0.25 \pm 0.12$ & $0.27 \pm 0.10$ & $0.31 \pm 0.03$ \\
\hline Total tocopherol $(\mu \mathrm{g} / \mathrm{g})$ & $25 \pm 5$ & $24 \pm 3$ & $24 \pm 3$ \\
\hline
\end{tabular}

Data are represented as mean $\pm S D, n=5$.

Table 5. Fatty acid composition of the fillet at the end of feeding.

\begin{tabular}{|c|c|c|c|}
\hline \multirow{3}{*}{ Fatty acids } & \multicolumn{3}{|c|}{ Treatment group } \\
\hline & Control & S-PC & S-PE \\
\hline & \multicolumn{3}{|c|}{$(g / 100 g)$} \\
\hline $14: 0$ & $4.0 \pm 0.3$ & $4.1 \pm 0.2$ & $3.7 \pm 0.2$ \\
\hline $16: 0$ & $21.2 \pm 1.4$ & $21.7 \pm 1.6$ & $21.7 \pm 0.7$ \\
\hline $16: 1$ & $7.2 \pm 1.0$ & $7.4 \pm 1.1$ & $7.5 \pm 1.1$ \\
\hline $18: 0$ & $5.0 \pm 0.2$ & $4.3 \pm 2.1$ & $4.9 \pm 0.4$ \\
\hline $18: 1$ & $26.9 \pm 1.1$ & $26.6 \pm 2.6$ & $25.4 \pm 0.8$ \\
\hline $18: 2(n-6)$ & $11.4 \pm 0.5$ & $9.2 \pm 0.8$ & $10.9 \pm 0.9$ \\
\hline $18: 3(n-6)$ & $0.1 \pm 0.1$ & $0.1 \pm 0.1$ & $0.1 \pm 0.1$ \\
\hline $18: 3(n-3)$ & $1.3 \pm 0.1$ & $1.3 \pm 0.1$ & $1.2 \pm 0.1$ \\
\hline $20: 1$ & $6.1 \pm 0.7$ & $6.3 \pm 0.5$ & $5.8 \pm 0.4$ \\
\hline $20: 2(n-6)$ & $0.6 \pm 0.1$ & $0.7 \pm 0.1$ & $0.6 \pm 0.1$ \\
\hline $20: 3(n-6)$ & $0.4 \pm 0.1$ & $0.4 \pm 0.1$ & $0.3 \pm 0.1$ \\
\hline $20: 4(n-6)$ & $0.6 \pm 0.2$ & $0.7 \pm 0.1$ & $0.6 \pm 0.1$ \\
\hline $22: 1$ & $3.6 \pm 0.2$ & $3.9 \pm 0.4$ & $3.4 \pm 0.2$ \\
\hline $20: 5(n-3)$ & $2.4 \pm 0.2$ & $2.8 \pm 0.3$ & $2.6 \pm 0.3$ \\
\hline $24: 0$ & $0.3 \pm 0.2$ & $0.3 \pm 0.2$ & $0.2 \pm 0.0$ \\
\hline $24: 1$ & $0.4 \pm 0.2$ & $0.4 \pm 0.2$ & $0.4 \pm 0.1$ \\
\hline $22: 5(n-3)$ & $0.6 \pm 0.2$ & $0.9 \pm 0.1$ & $0.8 \pm 0.1$ \\
\hline $22: 6(n-3)$ & $7.4 \pm 1.0$ & $8.5 \pm 1.6$ & $8.9 \pm 1.6$ \\
\hline
\end{tabular}

Data are represented as mean $\pm \mathrm{SD}, \mathrm{n}=5$.

test, the TBARS level was increased to more than double in all groups, and the level was significantly lower in the S-PC (120.9 nmol / g) and S-PE (111.2 nmol / g) groups than in the Control group (157.4 nmol / g) (Fig. 2).

The fatty acid composition of the fish fillet phospholipids was analyzed after completion of the feeding experiment

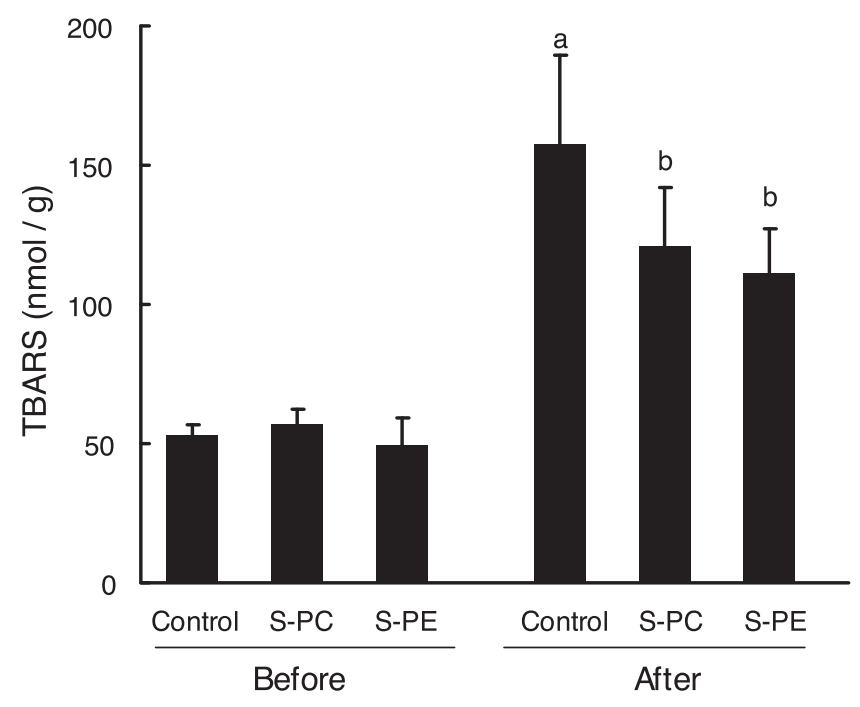

Fig. 2. Effects of dietary phospholipids on thiobarbituric acid reactive substances (TBARS) in rainbow trout fillets before and after the oxidation test. Data are represented as mean $\pm \mathrm{SD}(\mathrm{n}=8)$. Letters indicate statistically significant differences $(P<0.05)$.

(Table 6). The analysis revealed that the ratios of 14:0, 16:0, 22:1 and 24:1 in the S-PE group were significantly lower than those of the control group, and 18:1 in the S-PC and S-PE groups were significantly lower than that of the control group. The ratios of 20:5 and 22:5 in the S-PC and S-PE groups were significantly higher than in the control group, and 22:6 in the S-PE group was higher than in the control group. The ratios of saturated and monounsaturated fatty acids were high in the Control group, while the ratio of PUFAs was high in the S-PC and S-PE groups. The ratios of n-3 PUFAs were especially high in the S-PC and S-PE groups. However, the ratios of n-6 PUFAs were not different. No significant differences were present in any fatty acid between the S-PC and S-PE groups.

Fatty acids bound to PC and PE were analyzed. Signifi- 
Table 6. Fatty acid composition of the fillet phospholipids at the end of the feeding.

\begin{tabular}{|c|c|c|c|}
\hline & \multicolumn{3}{|c|}{ Treatment group } \\
\hline & Control & S-PC & S-PE \\
\hline & \multicolumn{3}{|c|}{$(\mathrm{g} / 100 \mathrm{~g})$} \\
\hline $14: 0$ & $3.3 \pm 0.2$ & $3.3 \pm 0.5^{\mathrm{a}}$ & $2.8 \pm 0.4^{b}$ \\
\hline $15: 0$ & $0.4 \pm 0.0$ & $0.3 \pm 0.1$ & $0.3 \pm 0.0$ \\
\hline $16: 0$ & $31.0 \pm 3.0^{\mathrm{a}}$ & $27.9 \pm 3.7^{\mathrm{ab}}$ & $25.6 \pm 1.9^{b}$ \\
\hline $16: 1$ & $3.7 \pm 1.4$ & $3.9 \pm 0.8$ & $4.0 \pm 0.5$ \\
\hline $17: 0$ & $0.4 \pm 0.0$ & $0.3 \pm 0.1$ & $0.3 \pm 0.0$ \\
\hline $18: 0$ & $8.0 \pm 0.9$ & $7.5 \pm 0.6$ & $6.0 \pm 0.3$ \\
\hline $18: 1$ & $20.1 \pm 1.1^{\mathrm{a}}$ & $18.5 \pm 1.0^{b}$ & $17.4 \pm 1.6^{b}$ \\
\hline $18: 2(n-6)$ & $6.5 \pm 0.6$ & $7.0 \pm 0.5$ & $6.3 \pm 0.2$ \\
\hline $18: 3(n-6)$ & $0.2 \pm 0.1$ & $0.2 \pm 0.0$ & $0.2 \pm 0.0$ \\
\hline $18: 3(n-3)$ & $0.7 \pm 0.1$ & $0.7 \pm 0.1$ & $0.7 \pm 0.1$ \\
\hline $20: 1$ & $2.6 \pm 0.1$ & $2.6 \pm 0.1$ & $2.3 \pm 0.3$ \\
\hline $20: 2(n-6)$ & $0.6 \pm 0.0$ & $0.7 \pm 0.1$ & $0.6 \pm 0.0$ \\
\hline $20: 3(n-6)$ & $0.6 \pm 0.3$ & $0.8 \pm 0.1$ & $0.7 \pm 0.1$ \\
\hline $20: 4(n-6)$ & $1.0 \pm 0.1$ & $1.0 \pm 0.3$ & $1.2 \pm 0.2$ \\
\hline $20: 3(n-3)$ & $0.1 \pm 0.0$ & $0.1 \pm 0.0$ & $0.1 \pm 0.0$ \\
\hline $22: 1$ & $1.3 \pm 0.1^{\mathrm{a}}$ & $1.4 \pm 0.1^{\mathrm{a}}$ & $1.2 \pm 0.2^{b}$ \\
\hline $20: 5(n-3)$ & $2.8 \pm 0.8^{\mathrm{a}}$ & $3.9 \pm 0.8^{b}$ & $4.4 \pm 0.7^{b}$ \\
\hline $24: 1$ & $0.8 \pm 0.1^{\mathrm{a}}$ & $0.5 \pm 0.3^{\mathrm{ab}}$ & $0.6 \pm 0.0^{b}$ \\
\hline $22: 5(n-3)$ & $0.9 \pm 0.2^{\mathrm{a}}$ & $1.1 \pm 0.2^{b}$ & $1.2 \pm 0.1^{b}$ \\
\hline $22: 6(n-3)$ & $13.2 \pm 3.7^{\mathrm{a}}$ & $16.1 \pm 4.9^{\mathrm{ab}}$ & $22.1 \pm 3.2^{b}$ \\
\hline
\end{tabular}

Data are represented as mean $\pm \mathrm{SD}, \mathrm{n}=5$. Letters indicate statistically significant differences $(P<0.05)$.

cant differences were noted in the fatty acid composition of phospholipids, and we focused on the highly abundant fatty acids $(16: 0,18: 1,20: 5$, and 22:6). To identify the binding positions of these fatty acids, $s n-1$ and $s n-2$ positions were individually analyzed. More than half of the $s n-1$ positions in fish fillet PC were bound by 16:0 fatty acid, with no significant difference among the diet groups. Significant differences were noted in the 20:5 and 22:6 fatty acid compositions, but their binding rates were extremely low (Fig. 3A). The $s n-2$ positions in PC were bound by 18:1, 20:5 and 22:6 at a high rate (Fig. 3B). The 20:5 and 22:6 rates were higher in the S-PC and S-PE groups than in the Control group. The 18:1 rate was lower in the S-PE than in the Control group. Regarding $s n-1$ positions in fish fillet PE, a significant difference was noted only in the rate of 20:5 bound to $s n-1$ position in PE between the Control and S-PC groups (Fig. 4A).
At the $s n-2$ positions in PE, the 18:1-bound rate was lower in the S-PC and S-PE groups than in the Control group, while the 22:6-bound rate was higher than in the Control group (Fig. 4B).

\section{Discussion}

This study showed that the main components of soybean phospholipids, soybean PC and soybean PE, increased the storage stability of fish fillets. When fish were fed soybean phospholipids, the rates of DHA bound to PC and PE increased in fish fillets.

Soybean phospholipids are known to exhibit an ex-vivo antioxidative effect on oil and fat. Zambiazi and Pryzybylski (1998) reported that the addition of 3\% or more phospholipids to vegetable oil was effective. Judde et al. (2003) showed that the addition of $1 \%$ soybean phospholipids inhibited oxidation in various oils, such as rapeseed, sunflower, soybean, walnut, palm, and fish oil, by measuring rancimat induction time $\left(110^{\circ} \mathrm{C}\right)$ and peroxide value $\left(40^{\circ} \mathrm{C}\right)$. The antioxidative mechanisms of phospholipids have been proposed to be due to the amino function of PC, PE and PS, which have been shown to have metal-chelating properties (Judde et al., 2003). Kashima et al. (1991) and Hamilton et al. (1998) reported that soybean PC and soybean PE increased the oxidative stability of soybean oil. Takeuchi et al. (1997) reported that the addition of soybean PE synergistically inhibited oxidation of n-3 PUFA with tocopherol. Recently, we reported that the oral administration of soybean phospholipids increased the oxidative stability of the meat in administered animals (Murano et al., 2008).

The oxidative stability of muscle food varies depending on the fatty acid composition and content of antioxidative substances. Morrissey et al. (1998) reported that oxidative stability was high when the PUFA content of meat was low. However, there was no significant difference in the fatty acid composition of fish fillets among the groups after the feeding period. The increased storage stability of meat with a high tocopherol or phospholipid content has been reported (Kim et al., 2006; Yamaguchi and Toyomizu, 1984), but no significant difference was noted in the tocopherol and phospholipid content. Based on these findings, enhanced fish fillet storage stability by dietary soybean phospholipids cannot be explained by the reduction in the PUFA content of fish fillets or increases in the tocopherol and phospholipid contents.

We analyzed the fatty acid composition of fish fillet phospholipids and identified differences in the composition of fatty acids, such as 16:0, 18:1, 20:5, and 22:6. Thus, we analyzed these fatty acid compositions in the main phospholipid species, PC and PE, and found that the 20:5 and 22:6 contents were high in the S-PC and S-PE groups. We also 

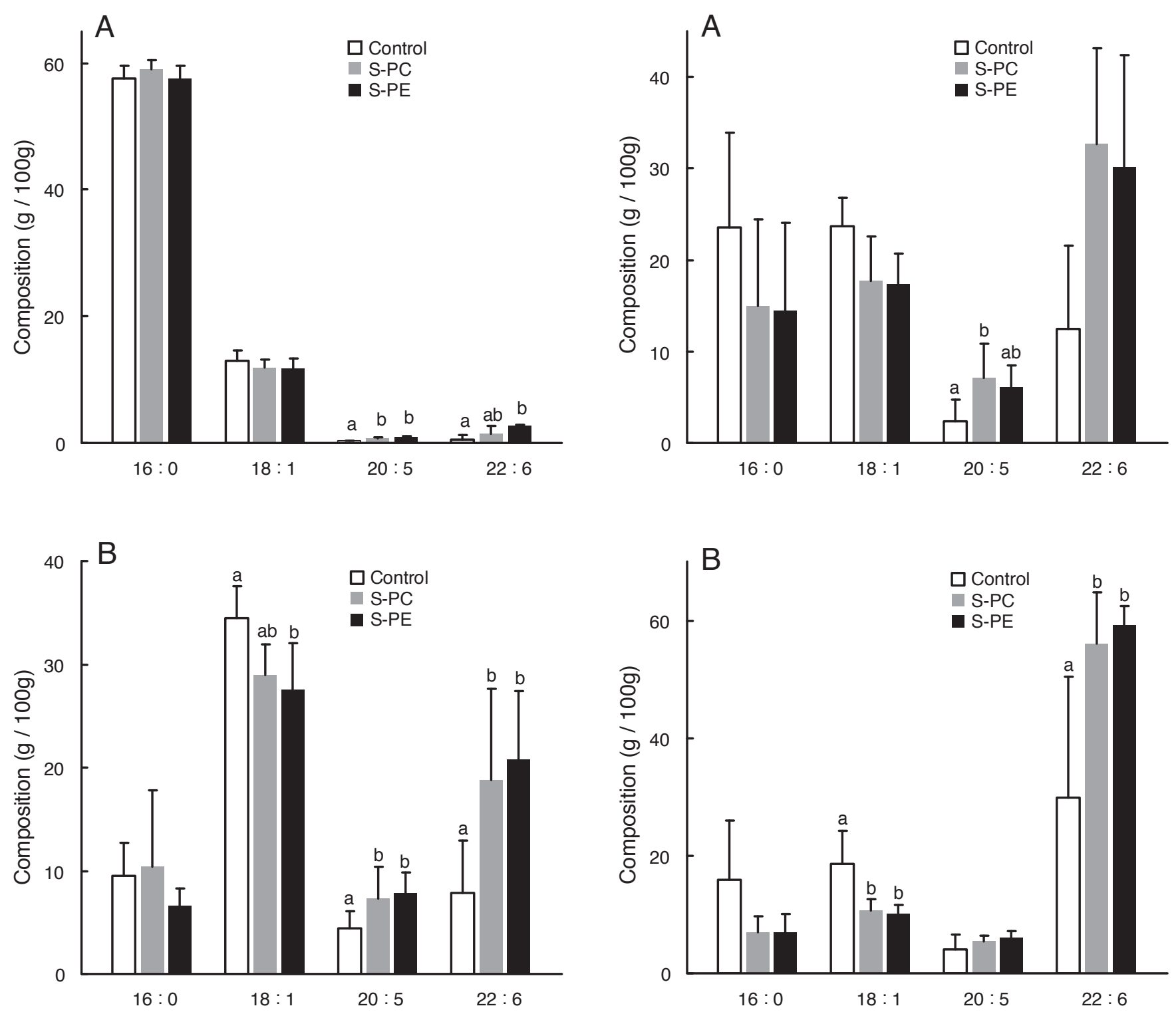

Fig. 3. Fatty acid composition of phosphatidylcholine at the sn1(A) and $s n-2$ (B) positions in the fillets at the end of feeding. Data are represented as mean $\pm \mathrm{SD}(\mathrm{n}=5)$. Letters indicate statistically significant differences $(P<0.05)$.

analyzed the amount of fatty acids bound to $s n-1$ and $s n-2$ positions in PC and PE. In both PC and PE, the $s n$-2-bound 22:6 content was significantly higher in the S-PC and S-PE groups. PC and PE are the main biomembrane components, and the increased oxidative stability of biomembranes by DHA-bound phospholipids has been reported. Lyberg et al. (2005) and Vikbjerg et al. (2007) showed, using a biomembrane model, that the storage stability of DHA bound to $s n-2$ positions of PC was increased. It has been clarified that PC containing DHA at $s n-2$ positions shows a variety of morphologies in the lipid bilayer, and is more dynamic than other fatty acid residues. Gawrisch et al. (2003) reported that DHA chains bend, tilt and back-fold. Therefore, DHA chains in membranes are, on average, much shorter

Fig. 4. Fatty acid composition of phosphatidylethanolamine at the $s n-1$ (A) and $s n-2$ (B) positions in the fillets at the end of feeding. Data are represented as mean $\pm \mathrm{SD}(\mathrm{n}=5)$. Letters indicate statistically significant differences $(P<0.05)$.

than DHA chains in extended conformations such as angleion or helical. Feller et al. (2001) reported that the presence of polyunsaturation in a hydrocarbon chain intrinsically increases the amount of conformational space accessible about the central methylene group as compared to saturated hydrocarbon chains. They showed a preferred model of extreme flexibility for the DHA chain, as opposed to one rigid structure or a few selected structures. The polyunsaturated chains rapidly convert between a large number of conformers when located in the lipid matrix. Accordingly, a gap is produced in the membrane, and a water molecule can easily enter near the double bond of DHA (Huster et al., 1997; Saiz and Klein, 2001). When a water molecule is present near this double bond, chain oxidation reactions may not easily occur 
because of steric interference by the water molecule (Miyashita, 2006). Regarding PE, the increased oxidative stability of a bilayer by DHA-bound PE has been reported. Lyberg et al. (2005) showed, using an emulsion system, that oxidative stability was higher when PE was bound by DHA than by other fatty acids. In the S-PC and S-PE groups, the oxidative stability of the biomembrane may be increased, which may have maintained the biomembrane function and antioxidative defense system, increasing the storage stability of fish fillets. Biomembrane deterioration is considered to be a general mechanism that markedly reduces the quality of muscle food (Stanley, 1991). N-3 PUFA contained in biomembranes are easily affected by radicals, and which triggers lipid peroxide production. The lipid peroxide produced induces further oxidation of membrane lipids, facilitating the deterioration of meat quality, as characterized by odor production, discoloration, and impaired texture (Stanley, 1991).

Takenaka et al. (2007) reported on the effect of PE, having saturated and unsaturated fatty acids, on lipid oxidation. They demonstrated that under the presence of $\alpha$-tocopherol, unsaturated PE has a synergistic effect. In the present study, the TG oxidation in fish fillets might be inhibited by the synergistic effect of unsaturated PE in combination with $\alpha$-tocopherol. Therefore, it would be of interest to investigate extracted lipids from fish fillets administered soybean phospholipids.

The PC- and PE-bound EPA contents were also increased by dietary high-purity phospholipids. Since only limited studies on the oxidative stability of EPA-bound phospholipids have been performed, the degree of contribution of an increase in the phospholipid-bound EPA content on the storage stability of fish fillets is unclear. The fatty acid composition in fish fillet total lipids was not affected by dietary highpurity phospholipids, and this was not contradictory to the findings of the previous report (Murano et al., 2008). Feeding phospholipids increased the PC- and PE-bound DHA and EPA contents of fish fillets, and these were not due to the dietary fatty acid compositions, because the DHA and EPA contents of the diets were equivalent.

To identify the active component of soybean phospholipids involved in improving the storage stability of fish fillets, rainbow trout were fed purified diets containing 1\% soybean PC and soybean PE with purities greater than $99 \%$ and $98 \%$, respectively, for 4 weeks. The storage stability of fillets from fish fed the diets containing high-purity phospholipids was greater than that of the Control group; showing that both soybean PC and soybean PE were components that increased storage stability. In fillets of fish fed the high-purity soybean PC and PE, DHA-binding to PC and PE, at the $s n-2$ position was significantly increased. These results indicate that the high phospholipid-bound DHA content has been one factor leading to the increased storage stability of fish fillets.

\section{References}

Connor, W.E. (2000). Importance of n-3 fatty acids in health and disease. Am. J. Clin. Nutr., 71, 171S-175S.

Feller, S.C., Gawrisch, K. and MackKerell, Jr.A.D. (2002). Polyunsaturated fatty acids in lipid bilayers: intrinsic and environmental contributions to their unique physical properties. J. Am. Chem. Soc., 124, 318-326.

Folch, J., Lees, M. and Sloane-stanley, G.H. (1957). A simple method for the isolation and purification of total lipids from animal tissue. J. Biol. Chem., 226, 497-506.

Gawrisch, K., Eldho, N.V. and Holte, L.L. (2003). The structure of DHA in phospholipid membranes. Lipids, 38, 445-452.

Hamilton, R.J., Kalu, C., McNeill, G.P. Padley, F.B. and Pierce, J.H. (1998). Effects of tocopherols, ascorbyl parmitate, and lecithin on autooxidation of fish oil. J. Am. Oil Chem. Soc., 75, 813-822.

Huang, C.J. and Shaw, H.M. (1994). Tissue vitamin E status is compromised by dietary protein insufficiency in young growing rats. $J$. Nutr., 124, 571-579.

Huster, D., Jim, A.J., Arnold, K. and Gawrisch, K. (1997). Water permiability of polyunsaturated lipid membranes measured by ${ }^{17}$ O NMR. Biophys. J., 73, 855-864.

Japan Oil Chemists' Society. (2003). Standard methods for the analysis of fats, oil and related materials. Method No. 2.4.11. JOCS, Tokyo, Japan (in Japanese).

Judde, A., Villeneuve, P., Rossignol-Castera, A. and Guillou, A.L. (2003). Antioxidant effect of soy lecithins on vegetable oil stability and their synergism with tocopherols. J. Am. Oil Chem. Soc., 80, 1209-1215.

Kashima, M., Cha, G.S., Isoda, Y., Hirano, J. and Miyazawa, T. (1991). The antioxidant effects of phospholipids on perilla oil. $J$. Am. Oil Chem. Soc., 68, 119-122.

Kaushik, S.J., Gouillou-Coustans, M.F. and Cho, C.Y. (1998). Application of the recommendations on vitamin requirements of finfish by NRC (1993) to salmonids and sea bass using practical and purified diets. Aquaculture, 161, 463-474.

Kim, B.C., Ryu, Y.C., Cho, Y.J. and Rhee, M.S. (2006). Influence of dietary $\alpha$-tocopheryl acetate supplementation on cholesterol oxidation in retail packed chicken meat during refrigerated storage. Biosci. Biotechnol. Biochem., 70, 808-814.

Lyberg, A.M., Fasoli, E. and Adlercreutz, P. (2005). Monitoring the oxidation of docosahexaenoic acid in lipids. Lipids, 40, 969-979.

Meynier, A., Genot, C. and Gandemer, G. (1999). Oxidation of muscle phospholipids in relation to their fatty acid composition with emphasis on volatile compounds. J. Sci. Food Agric., 79, 797-804.

Miyashita, K. (2006). Oxidative stability of marine lipids in aqueous solution. Nippon Suisan Gakkaishi, 72, 636-639 (in Japa- 
nese).

Morrissey, P.A., Sheehy, P.J.A., Galvin, K., Kerry, J.P. and Buckeley, D.J. (1998). Lipid stability in meat and meat products. Meat Sci., 49, S73-S86.

Murano, Y., Funabashi, T., Sekine, S., Aoyama, T. and Takeuchi, H. (2007). Effect of dietary lard containing higher $\alpha$-linolenic acid on plasma triacylglycerol in rats. J. Oleo Sci., 56, 361-367.

Murano, Y., Funabashi, T., Sekine, S. and Takeuchi, H. (2008). Improvement in storage stability of fish fillet using dietary soybean phospholipids. Food Sci. Technol. Res., 14, 55-61.

Ogino, C., Takeuchi, L., Takeda, H. and Watanabe, T. (1979). Availability of dietary phosphorus in carp and rainbow trout. Bull. Japan. Soc. Sci. Fish., 45, 1527-1532 (in Japanese).

Olbrich, K., Rawicz, W., Needham, D. and Evans, E. (2000). Water permeability and mechanical strength of polyunsaturated lipid bilayers. Biophys. J., 79, 321-327.

Saiz, L. and Klein, M.L. (2001). Structural properties of a highly polyunsaturated lipid bilayer from molecular dynamics simulations. Biophys. J., 81, 204-216.

Shantha N. C. and Ackman R.G. (1990) . Nervonic acid versus tricosanoic acid as internal standards in quantitative gas chromatographic analyses of fish oil longerchain n-3 polyunsaturated fatty acid methyl esters. J. Chromatograph., 533, 1-10.

Schneider, M. (2006). Phospholipids - what's new?. Lipid Technol., 18, 250-255.

Stanley, D.W. (1991). Biological membrane deterioration and associated quality losses in food tissues. Crit. Rev. Food Sci. Nutr.,
30, 487-553.

Takenaka, A., Hosokawa, M. and Miyashita, K. (2007). Unsaturated phosphatidylethanolamine as effective synergist in combination with $\alpha$-tocopherol. J. Oleo Sci., 56, 511-516.

Takeuchi, M. Hara, S. Hibino, H. Tanaka, Y. and Totani, Y. (1997). Autooxidative behavor of polyunsaturated phospholipids. III Comparison of autooxidative behavor between polyunsaturated phospholipid and triacylglycerol. Nihon Yukagakkaishi, 46 1489-1494.

Takeuchi, H., Sakurai, C., Noda, R., Sekine, S., Murano, Y., Wanaka, K., Kasai, M., Watanabe, S., Aoyama, T. and Kondo, K. (2007). Antihypertensive effect and safety of dietary $\alpha$-linolenic acid in subjects with high-normal blood pressure and mild hypertension. J. Oleo Sci., 56, 347-360.

Vikbjerg, A.F., Andresen, T.L., Jørgensen, K. Mu, H. and Xu, X. (2007). Oxidative stability of liposomes composed of docosahexaenoic acid-containing phospholipids. J. Am. Oil Chem. Soc., 84, 631-637.

Wells, M.A. and Hanahan, D.J. (1969). Methods in Enzymology. Academic Press, New York., 14, pp. 178-184.

Yamaguchi, K. and Toyomizu, M. (1984). A role of phospholipid in antioxygenic action of lipids from the ordinary muscle of lean fish. Bull. Japan. Soc. Sci. Fish., 50, 1897-1903 (in Japanese).

Zambiazi, R.C. and Przybylski, R. (1998). Effect of endogenous minor components on the oxidative stability of vegetable oils. Lipid Technol., 10, 58-62. 